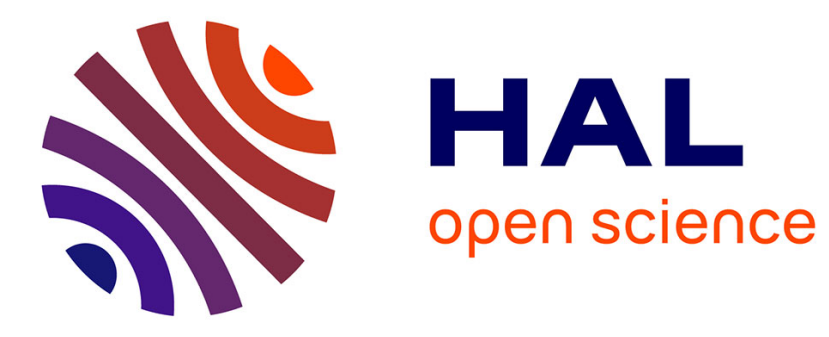

\title{
Embryonal tumors with multilayered rosettes in children: the SFCE experience
}

Meryl Horwitz, Christelle Dufour, Pierre Leblond, Franck Bourdeaut, Cécile Faure-Conter, Anne-Isabelle Bertozzi, Bernadette Delisle, Gilles Palenzuela, Anne Jouvet, Didier Scavarda, et al.

\section{To cite this version:}

Meryl Horwitz, Christelle Dufour, Pierre Leblond, Franck Bourdeaut, Cécile Faure-Conter, et al.. Embryonal tumors with multilayered rosettes in children: the SFCE experience. Child's Nervous System, 2016, 32 (2), pp.299-305. 10.1007/s00381-015-2920-2 . hal-01368643

\section{HAL Id: hal-01368643 \\ https://hal-amu.archives-ouvertes.fr/hal-01368643}

Submitted on 12 Oct 2016

HAL is a multi-disciplinary open access archive for the deposit and dissemination of scientific research documents, whether they are published or not. The documents may come from teaching and research institutions in France or abroad, or from public or private research centers.
L'archive ouverte pluridisciplinaire HAL, est destinée au dépôt et à la diffusion de documents scientifiques de niveau recherche, publiés ou non, émanant des établissements d'enseignement et de recherche français ou étrangers, des laboratoires publics ou privés.

\section{(이) $\$$}

Distributed under a Creative Commons Attribution - NonCommercial - NoDerivatives 4.0 


\title{
Embryonal tumors with multilayered rosettes in children: the SFCE experience
}

\author{
Meryl Horwitz ${ }^{1}$ - Christelle Dufour ${ }^{2}$ - Pierre Leblond ${ }^{3}$ - Franck Bourdeaut ${ }^{4,5}$. \\ Cécile Faure-Conter ${ }^{6}$ • Anne-Isabelle Bertozzi ${ }^{7}$ - Marie Bernadette Delisle ${ }^{8}$. \\ Gilles Palenzuela $^{9}$ • Anne Jouvet ${ }^{10}$ - Didier Scavarda ${ }^{11}$ - Matthieu Vinchon ${ }^{12}$. \\ Laetitia Padovani $^{13}$ • Jean Gaudart ${ }^{14,15}$ • Dominique Figarella Branger ${ }^{16}$. \\ Nicolas Andre $1,17,18$
}

Received: 22 June 2015 / Accepted: 23 September 2015 /Published online: 5 October 2015

(C) Springer-Verlag Berlin Heidelberg 2015

\begin{abstract}
Purposes The purpose of this study was to retrospectively study embryonal tumors with multilayered rosettes (ETMR), a rare new entity that gathers ETAN-TR (embryonal tumor with abundant neuropil and true rosettes), ependymoblastomas, and
\end{abstract}

Nicolas Andre

nicolas.andre@ap-hm.fr

Meryl Horwitz

meryl.horwitz@gmail.com

Christelle Dufour

christelle.dufour@gustaveroussy.fr

Pierre Leblond

p-leblond@o-lambret.fr

Franck Bourdeaut

franck.bourdeaut@curie.fr

Cécile Faure-Conter

cecile.conter@ihope.fr

Anne-Isabelle Bertozzi

bertozzi.ai@chu-toulouse.fr

Marie Bernadette Delisle

delisle.b@chu-toulouse.fr

Gilles Palenzuela

gillespalen@yahoo.fr

Anne Jouvet

anne.jouvet@chu-lyon.fr

Didier Scavarda

didier.scavarda@ap-hm.fr

Matthieu Vinchon

matthieu.vinchon@chru-lille.fr

Laetitia Padovani

laetitia.padovani@ap-hm.fr medulloepitheliomas, in order to improve their descriptions and try to better define therapeutic modalities.

Methods Patients with ETMR, ETAN-TR, ependymoblastoma, and medulloepithelioma treated in SFCE centres (Société Française de lutte contre les Cancers et les leucémies de l'Enfant

Jean Gaudart

jean.gaudart@ap-hm.fr

Dominique Figarella Branger

dominiqueFrance.figarella@ap-hm.fr

1 Department of Pediatric Hematology-Oncology, APHM, La Timone Hospital, 264 rue Saint-Pierre, 13005, Marseille, France

2 Department of Pediatric and Adolescent Oncology, Gustave Roussy Campus, 114, rue Edouard-Vaillant, 94805 Villejuif Cedex, France

3 Department of Pediatric Oncology, Oscar Lambret, 3 rue Frederic Combemale, BP 307, 59020 Lille Cedex, France

4 Department of Pediatric Oncology, Institut Curie, 26, rue d'Ulm, 75005 Paris, France

5 INSERM U830, Laboratory of Genetics and Cancer Biology, Institut Curie, Paris, France

6 Department of Pediatrics, Centre Léon Bérard, 28 Prom. Léa et Napoléon Bullukian, 69008 Lyon, France

7 Department of Hematology-Oncology, Children University Hospital, 330 Avenue de Grande Bretagne, 31300 Toulouse, France

8 Department of Neuropathology, Toulouse Rangueil University Hospital, 1, avenue du Pr Jean Poulhès, 31400 Toulouse, France

9 Department of Pediatric Hematology-Oncology, University Hospital of Montpellier, 191, avenue du doyen Gaston Giraud, 34295 Montpellier cedex, France

10 Department of Pathology and neuropathology, "Pierre Werthmeier" Hospital, Boulevard Pinel, 69677 Bron, France 
et de l'adolescent) since 2000 were collected. Data were retrieved from clinical charts.

Results Thirty-eight patients were included in the analysis. Seventeen had an ETAN-TR, 13 had a medulloepithelioma, and 8 had an ETMR. No ependymoblastoma was included. The median age at diagnosis was 31 months (range, 2.8141 months). The predominant tumor location was supratentorial ( $66 \%) ; 18.4 \%$ patients had metastatic lesion.

LIN28A expression was positive in $11 / 11$ patients. Amplification of the locus $19 \mathrm{q} 13.42$ was positive in $10 / 12$ patients. Thirty patients were treated according to the primitive neuroectodermal tumors of high risk (PNET-HR) protocol. The median time of follow-up was 0.9 years (range 0.1 to 15.3 years). The 1-year event-free survival (EFS) and overall survival (OS) were, respectively, $36 \%$ CI $95 \%$ (23-55) and $45 \%$ CI $95 \%$ (31-64). On multivariate analysis, complete surgical resection, radiotherapy, and high-dose chemotherapy were associated with a better overall survival with a relative risk of, respectively, 7.9 CI $95 \%(2.6-23.5) p<0.0002,41.8$ CI $95 \%(9.4-186)$ $p<0.0001$, and 3.5 CI $95 \%(1.3-9.5) p=0.012$.

Conclusion Prognosis of ETMR remains dismal despite multimodal therapy. LIN28A immunostaining and 19q13.42 amplification should be systematically done to secure the diagnosis. Complete surgical resection, radiotherapy, and highdose chemotherapy are associated with better outcome.

Keywords Brain tumors · Children · Radiotherapy · Surgery · Chemotherapy $\cdot$ ETMR

\section{Introduction}

Embryonal tumors with neuropil and true rosettes abundant (ETAN-TR), ependymoblastomas, and medulloepitheliomas are three distinct pathologies which actually represent

11 Department of Neurosurgery, APHM, La Timone Hospital, 264 rue Saint-Pierre, 13005 Marseille, France

12 Department of Pediatric Neurosurgery, Lille University Hospital, 2 Avenue Oscar Lambret, 59000 Lille, France

13 Department of Radiation Oncology and Pediatrics, APHM, La Timone Hospital, 264 rue Saint-Pierre, 13005 Marseille, France

14 BIOSTIC, Pôle de Santé Publique, AP-HM, Marseille, 264 rue Saint-Pierre, 13005 Marseille, France

15 UMR912 SESSTIM, Aix-Marseille University, Marseille, France

16 Department of Pathology, APHM, La Timone Hospital, Université Aix-Marseille, 264 rue Saint-Pierre, 13005 Marseille, France

17 INSERM UMR 911, Centre de Recherche en Oncologie biologique et en Oncopharmacologie, Aix-Marseille University, 264 rue Saint-Pierre, 13005 Marseille, France

18 Department of Pediatric Hematology and Oncology, Children Hospital of La Timone, 264, rue Saint-Pierre, 13005 Marseille, France different manifestations of the same histological and biological entity $[1,2]$. The term embryonal tumors with multilayered rosettes (ETMR) was proposed in 2010 as a unifying entity by Paulus and Kleihues [3] as the histological central element found in these tumors was the presence of multilayered rosettes. Moreover, ETMR seem to have a specific molecular signature with a positivity of LIN28A on immunohistochemistry and amplification of the locus 19q13.42 in FISH analysis [4, 5]. ETMR belongs to the group of the primitive neuroectodermal tumors of the central nervous system (PNET) according to the World Health Organization (WHO) classification [6].

ETMR are quite rare entities; their prevalence is not accurately estimated. They are likely under-diagnosed with only a few cases reported in the literature since 2000 [2,3, 7, 8]. They occur mostly in children aged less than 2 years old. In the literature, the localization of the majority of the primitive tumor is supratentorial with signs of increased intracranial pressure. Treatment of ETMR usually starts with maximal surgical resection. The role of adjuvant treatment with either chemotherapy and/or radiotherapy has not yet been well established for this disease.

To improve our knowledge about ETMR, we collected cases treated in SFCE centers (Société Française des Cancers et leucémies de l'Enfant et de l'adolescent) from January 2000 to May 2014 in order to retrospectively study clinical, radiological, biological, and prognostic descriptions to improve their descriptions and try to better define therapeutic modalities.

\section{Patients and methods}

\section{Study design, patients, data collection}

Patients aged less than 18 years with newly diagnosed ETMR who were treated in France between January 2000 and 1 May 2014 were identified retrospectively by consulting local oncologists' and pathologists' databases in SFCE centers using the following diagnosis: ETMR, ETAN-TR, ependymoblastoma, and medulloepithelioma. A standardized data sheet was sent to pediatric oncologists who were members of the SFCE to collect clinical data. The questionnaire included information about sex, age at time of presentation, duration of the symptoms at time of presentation, location of tumor, extent of resection (complete resection, partial resection, biopsy), pathological biological and molecular diagnosis, adjuvant therapies, and follow-up. At diagnosis, stage of disease was evaluated by initial cranial and spinal MRI and CSF cytology. The extent of initial resection was assessed by MRI or computed tomography performed within $72 \mathrm{~h}$ after surgery. Metastatic disease was assessed using the Chang staging system. Tumors were subjected to a central histopathological 
review by members of the national pediatric brain tumors board (GENOP). When it was feasible, immunohistochemistry analysis applying a LIN28A polyclonal antibody and array-based comparative genomic hybridization (CGHarray) was performed. The data were coded to ensure confidentiality. This study has been approved by the National Ethical Committee (CCTIRS).

\section{Statistical analysis:}

Relapses and deaths were considered as events. Event-free survival (EFS), overall survival (OS), and univariate analysis were estimated by the Kaplan-Meier method, and differences between groups were assessed by the log-rank test. Survival estimates referred to 1 and 3 years from diagnosis and the related $95 \%$ confidence intervals (95\% CI) were calculated. Cox model was used to calculate the multivariate analysis by a stepwise selection. Descriptive statistics were reported as absolute frequencies and percentages for qualitative data, while median and range were used for continuous variables. When performing univariate analysis, those variables were used: age $\leq 4$ years or $>4$ years, age $<4$ years or 4 to 5 years or 5 to 8 years or 8 to 11 years, histological diagnosis (ETMR, ETAN-TR, medulloepithelioma, ependymoblastoma), surgery, chemotherapy, radiotherapy, high-dose chemotherapy, and primitive neuroectodermal tumors of high risk (PNET-HR) (yes or no). Positive variables identified with univariate analysis were used in the multivaried analysis. All statistical tests were two sided and a " $p$ " value $<0.05$ was considered significant. Statistical analysis was performed using $\mathrm{R}$ version 3.0.2.

\section{Results:}

\section{Patients' characteristics}

Forty-two patients with an ETMR were identified in the SFCE centers, but 4 patients were excluded because data could not be retrieved or the diagnosis was uncertain. Thirty-eight patients were therefore analyzed. The median age at diagnosis was 31.1 months (range 2.8-141 months) and the sex ratio was 0.4 (male/female). Main clinical signs were a sided weakness $(34 \%)$ and increased intracranial pressure (53 \%). Confusion, seizures, and cerebellar syndrome occurred in 26, 21, and $21 \%$ of children, respectively. Torticollis occurred in $8 \%$ of cases and visual impairment was found in $11 \%$ of patients.

The predominant tumor location was supratentorial (66\%). Tumors were infratentorial in the others (26\%). One patient had a medullar lesion. Eighteen percent of patients had metastatic disease at initial presentation. In all cases, the metastasis was evidenced by MRI while metastatic cells were never found in the cerebrospinal fluid at the time of diagnosis.

\section{Histopathological characteristics}

A histopathological review was performed in $100 \%$ of the 38 patients. Noteworthy, 6 children had an uncorrect diagnosis (1 ependymoma, 5 PNET). Seventeen patients had ETAN-TR, 13 had medulloepithelioma, 8 had ETMR, and 0 had ependymoblastoma (Table 1). LIN28A expression was searched for 11 patients and was positive in $100 \%$ of the cases. The amplification of the locus $19 \mathrm{q} 13.42$, searched for in 12 children, was present in $83 \%$ of the patients. The gain of the chromosome 2 and other chromosome gains were searched for in 11 patients and were positive, respectively, in 27 and $54 \%$ of the cases. Data are summarized in Table 2.

\section{Treatment strategy and outcome}

All children but 8 were treated according to the PNET-HR protocol. The strategy relies on the combination of surgery, conventional chemotherapy ( 2 courses of etoposidecarboplatin), followed by sequential high-dose chemotherapy (melphalan/cisplatinum/melphalan/cisplatinum/thiotepa) each course being followed by peripheral blood stem cell transplantation and reduced dose radiation therapy [9]. Details of all the 38 patients are provided in Table 3.

All children received adjuvant chemotherapy, after a complete resection for 12 patients, or a partial resection/biopsy for 18 patients. Out of the 30 patients who received etoposide/ carboplatinum, 4 experienced disease progression (1 who had a complete resection and 3 who had a partial resection). In patients who underwent complete resection, 6 children received the complete PNET-HR protocol and 3 are alive with a follow-up of, respectively, 12, 8.6, and 3.6 years. Out of the patients who had a post-surgery residual disease (18 patients), only two completed the whole protocol PNET-HR. The remaining patients presented tumor progression and all patients but 2 died.

Among the patients who were not treated according the the PNET-HR protocol, one child with a localized ETMR underwent a complete removal of the tumor after 2 surgeries. CSF became positive after the second surgery. She was treated with temozolomide-irinotecan chemotherapy, followed by craniospinal radiotherapy and by a 1-year metronomic temozolomide-irinotecan maintenance. This child is alive and in CR with a follow-up of more than a year, without receiving high-dose chemotherapy. Three other children received chemotherapy with etoposide/carboplatin after a surgical resection (2 partial and 1 total) and radiotherapy after thiotepa. One of them received a maintenance treatment with temozolomide. Those children are alive with a follow-up of respectively $1,1.3$, and 5.8 years.

Of note, 6 tumors out of the 16 tumors of who received radiotherapy progressed and 10 children are alive. All patients 
Table 1 Patient characteristics

\begin{tabular}{|c|c|c|c|c|c|}
\hline & Total & ETANTR & MedulloeP & ETMR & $\mathrm{EP}$ \\
\hline Number (\%) & $38(100 \%)$ & $17(45 \%)$ & $13(34 \%)$ & $8(21 \%)$ & 0 \\
\hline Median age (months) (min-max) & $31(2.8-141)$ & $32.9(15.5-141)$ & $31.1(2.9-143.4)$ & $27.2(12.5-43.8)$ & \\
\hline Mean age (months) $(\sigma)$ & $40.8[6]$ & $33.4(9.5)$ & $60.3(53.5)$ & $27.3(10.5)$ & \\
\hline \multicolumn{6}{|l|}{ Sex } \\
\hline$\%$ Male & 26 & 70.6 & 84.6 & 62.5 & \\
\hline$\%$ Female & 72 & 29.4 & 15.4 & 37.5 & \\
\hline Sex ratio & 0.4 & 0.4 & 0.2 & 0.4 & \\
\hline Median time to diagnosis (days) (min-max) & $22(1-203)$ & $32(1-172)$ & $28(4-203)$ & $23.5(15-615)$ & \\
\hline \multicolumn{6}{|l|}{ Clinical signs, $\% *$} \\
\hline Sided weakness & 34 & 53 & 7.7 & 37.5 & \\
\hline Confusion & 26 & 23.5 & 30.8 & 25 & \\
\hline Increased IP & 53 & 53 & 69.2 & 25 & \\
\hline Seizures & 21 & 23.5 & 15.4 & 0 & \\
\hline Cerebellar syndrome & 21 & 23.5 & 15.4 & 25 & \\
\hline Torticolis & 8 & 0 & 15.4 & 12.5 & \\
\hline Visual impairment & 11 & 11.8 & 15.4 & 0 & \\
\hline Others & 3 & 5.9 & 7.7 & 12.5 & \\
\hline MV & 3 & 5.9 & 0 & 0 & \\
\hline \multicolumn{6}{|l|}{ Location, \% } \\
\hline Supratentorial & 66 & 76.4 & 53.8 & 62.5 & \\
\hline Infratentorial & 26.1 & 6 & 46.2 & 37.5 & \\
\hline Other & 7.9 & 17.6 & 0 & 0 & \\
\hline \multicolumn{6}{|l|}{ Metastatic CNS \% } \\
\hline Yes & 18 & 23.5 & 23.1 & 0 & \\
\hline No & 79 & 76.5 & 69.2 & 0 & \\
\hline MV & 2.6 & 0 & 7.7 & 0 & \\
\hline
\end{tabular}

$\sigma$ range, $M$ male, $F$ female, $M e d u l l o e P$ medulloepithelioma, $E P$ ependymoblastoma, $I P$ intracranial pressure, $C N S$ central nervous system, $M V$ missing values

*Total can be over $100 \%$ as one patient can present several clinical signs

Table 2 Histopathological and biomolecular characteristics

\begin{tabular}{ll}
\hline Histopathological review & \\
Number at initial diagnosis (\%) & $38(100 \%)$ \\
Number of changes in diagnosis (\%) & $6(16 \%)$ \\
LIN28 & \\
$\quad$ Number of patients analyzed (\%) & $11(29 \%)$ \\
Number of positive staining (\%) & $11(100 \%)$ \\
ampl_19q13.42 & \\
Number of patients analyzed (\%) & $12(32 \%)$ \\
Number of positive (\%) & $10(83 \%)$ \\
Gain of chromosome 2 & \\
$\quad$ Number of patients analyzed (\%) & $11(29 \%)$ \\
Number of positive (\%) & $3(27 \%)$ \\
Other chromosomics gains & \\
Number of patients analyzed (\%) & $11(29 \%)$ \\
Number of positive (\%) & $6(54 \%)$ \\
\hline
\end{tabular}

alive and in complete remission underwent complete surgical resection and received radiotherapy.

The median time of follow-up for the whole population was 0.9 years (range 0.1 to 15.3 years). The 1 -year eventfree survival (EFS) and overall survival (OS) rate were $36 \%$ CI $95 \%(23-55)$ and $45 \%$ CI $95 \%$ (31-64), respectively (Figs. 1 and 2). The median EFS and OS rate were 5.4 and 10.7 months, respectively. The median time to first progression/relapsed was 4.3 months (range, 0.944.8 months). Twenty-seven relapses were observed, $74 \%$ were localized, $22 \%$ were localized and metastatic, and $4 \%$ were only metastatic.

\section{Prognostic factors}

The univariate analysis indicates that complete surgical resection, radiotherapy, and high-dose chemotherapy were significantly associated with a better OS (respectively, $p<0.003, p<0.0001$, and $p<0.012$ ). Furthermore, 
Table 3 Treatment strategy and outcome

\begin{tabular}{|c|c|c|c|c|c|c|c|c|c|c|}
\hline Number & Sex & Age (year) & Location & CNS Meta & Surgery & Chemotherapy & $\begin{array}{l}\text { High-dose } \\
\text { chemotherapy }\end{array}$ & $\mathrm{RX}$ & Outcome & Last news (m) \\
\hline 1 & $\mathrm{~F}$ & 1.3 & Infra & Yes & Total & VP-Carbo & No & No & Death & 1.6 \\
\hline 2 & $\mathrm{~F}$ & 2.6 & Supra & No & Total & VP-carbo X2 & No & No & Death & 11.3 \\
\hline 3 & $\mathrm{~F}$ & 11.5 & Supra & No & Total & No & No & Yes & Alive, CR & 183.9 \\
\hline 4 & $\mathrm{~F}$ & 3.6 & Supra & No & Partial & TEMIRI X3/maintenance & No & Yes & Alive, CR & 13.4 \\
\hline 5 & $\mathrm{~F}$ & 11.8 & Infra & No & Total & VP-carbo X2 & Thio & Yes & Alive CR & 12.2 \\
\hline 6 & M & 3.6 & Infra & No & Partial & VP-carbo X2 & No & No & Death & 4.2 \\
\hline 7 & $\mathrm{~F}$ & 2.3 & Supra & No & Partial & VP-carbo X2 & No & No & Death & 4.3 \\
\hline 8 & M & 1.5 & Infra & No & Partial & VP-carbo X2 & Thio X2/TMZ & Yes & Alive, PR & 15.9 \\
\hline 9 & $\mathrm{~F}$ & 2.2 & Supra & No & Partial & VP-Carbo X2 & No & No & Death & 7.3 \\
\hline 10 & $\mathrm{~F}$ & 2.6 & Supra & No & Partial & VP-carbo X2 & $\mathrm{Mel} / \mathrm{Cis}$ & No & Death & 6.3 \\
\hline 11 & $\mathrm{~F}$ & 2.7 & Supra & No & Total & VP-carbo X2 & Mel-Cis X2 & No & Death & 7.6 \\
\hline 12 & M & 2.9 & Spinal cord & Yes & Total & VP-carbo X2 & Mel-Cis X2/Thio & Yes & Alive, CR & 43.2 \\
\hline 13 & $\mathrm{~F}$ & 2.2 & Supra & No & Total & VP-carbo/carbo & Mel-Cis X2/Thio & Yes & Alive, CR & 143.6 \\
\hline 14 & $\mathrm{~F}$ & 3.3 & Supra & No & Total & VP-carbo X2 & Mel/Cis/Mel/Thio & No & Death & 10.6 \\
\hline 15 & M & 1.6 & Supra & Yes & Partial & VP-carbo X2 & $\mathrm{Mel} / \mathrm{Cis}$ & No & Death & 7.8 \\
\hline 16 & $\mathrm{~F}$ & 1.3 & Supra & No & Partial & VP-carbo X2 & Mel/Thio & No & Death & 7.8 \\
\hline 17 & $\mathrm{~F}$ & 3 & Supra & No & Partial & VP-carbo X2 & $\mathrm{Mel} / \mathrm{Cis} / \mathrm{Thio} \mathrm{X} 2$ & Yes & Death & 16.9 \\
\hline 18 & M & 8.1 & Infra & No & Partial & VP-carbo X2 & $\mathrm{Mel} / \mathrm{Cis}$ & No & Death & 9.6 \\
\hline 19 & $\mathrm{~F}$ & 3.2 & Supra & No & Partial & VP-carbo X2 & $\mathrm{Mel} / \mathrm{Cis} / \mathrm{Thio}$ & Yes & Alive, PR & 90.9 \\
\hline 20 & $\mathrm{~F}$ & 6.8 & Supra & No & Total & VP-carbo X2 & Mel-Cis X2 & Yes & Alive, CR & 63.8 \\
\hline 21 & $\mathrm{~F}$ & 2 & Supra & No & Partial & VP-carbo X2 & Mel X2/Thio X2 & Yes & Alive, CR & 143.4 \\
\hline 22 & $\mathrm{~F}$ & 3.5 & Supra & No & Partial & VP-carbo X2 & $\mathrm{Mel} / \mathrm{Cis}$ & No & Death & 4.5 \\
\hline 23 & $\mathrm{~F}$ & 2.5 & Supra & Yes & Partial & VP-carbo X2 & Mel/Thio X2 & No & Death & 8 \\
\hline 24 & M & 1.3 & Supra + Infra & Yes & Partial & VP-carbo X2 & $\mathrm{Mel} / \mathrm{Cis} / \mathrm{Mel}$ & No & Death & 10.1 \\
\hline 25 & M & 2.6 & Infra & No & Biopsy & VP-carbo X2 & Mel & No & Death & 6.5 \\
\hline 26 & M & 1.4 & Infra & No & Total & VP-carbo X2 & Mel-Cis X2/Thio & Yes & Death & 21.2 \\
\hline 27 & $\mathrm{~F}$ & 3.2 & Supra & No & Partial & VP-carbo X2 & $\mathrm{Mel} / \mathrm{Cis}$ & No & Death & 8.3 \\
\hline 28 & $\mathrm{~F}$ & 2.6 & Infra & No & Total & VP-carbo X2 & Mel-Cis X2/Thio & Yes & Alive CR & 103.3 \\
\hline 29 & $\mathrm{~F}$ & 1.8 & Supra & No & Total & VP-carbo X2 & Mel-Cis X2/Thio & No & Death & 13 \\
\hline 30 & $\mathrm{~F}$ & 4.3 & Supra & No & Partial & VP-carbo X2 & $\mathrm{Mel} / \mathrm{Cis}$ & Yes & Death & 10 \\
\hline 31 & $\mathrm{~F}$ & 3.3 & Supra & No & Total & VP-carbo X2 & $\mathrm{Mel} / \mathrm{Cis}$ & No & Death & 19.2 \\
\hline 32 & $\mathrm{~F}$ & 0.2 & Supra & MV & Total & BBFSOP & No & No & Death & 9.2 \\
\hline 33 & M & 2 & Supra + Infra & Yes & Biopsy & VP-carbo X2 & $\mathrm{Mel} / \mathrm{Cis}$ & No & Death & 5.3 \\
\hline 34 & $\mathrm{~F}$ & 11.6 & Infra & Yes & Total & VP-carbo X2 & Thio X2/TMZ & Yes & Alive CR & 70.5 \\
\hline 35 & $\mathrm{~F}$ & 1.8 & Supra & No & Total & VP-Carbo X2 & Mel-Cis X2/Thio & Yes & Death & 29 \\
\hline 36 & $\mathrm{~F}$ & 3.3 & Supra & No & Partial & MV & MV & Yes & Death & 11.9 \\
\hline 37 & $\mathrm{~F}$ & 1 & Infra & No & Partial & VP-Carbo X2 & Mel & No & Death & 6 \\
\hline 38 & M & 2.3 & Supra & No & Total & VP-Carbo X2 & $\mathrm{Mel} / \mathrm{Cis}$ & No & Death & 13 \\
\hline
\end{tabular}

$N$ number, $F$ female, $M$ male, Supra supratentorial, Infra infratentorial, Meta metastasis, $M V$ missing values, $V P$ etoposide, carbo carboplatin, TEMIRI temozolomide-irinotecan, $T M Z$ temozolomide, BBSFOP 2 cycles of Paraplatine + Natulan then Vepeside + Cisplatinum then vincristine + cyclophosphamide, $M e l$ Melphalan, $C i s$ cisplatinum, Thio thiotepa, $R x$ radiotherapy, $C R$ complete remission, $P R$ partial remission, $y$ years, $m$ months

according to the multivariate analysis, radiotherapy with a relative risk (RR) of 41.8 CI $95 \%(9.4-186) p<0.0001$, surgical complete resection with a 7.9 CI $95 \%(2.6-23.5)$ $p<0.0002$, and high-dose chemotherapy with a RR $3.5 \mathrm{CI}$ $95 \%(1.3-9.5) p=0.012$ were prognostic factors associated with a better OS.

\section{Discussion}

ETMR is a new entity, which encompasses ETAN-TR, ependymoblastomas, medulloepitheliomas. Indeed, those tumors seem to present clinical, histopathological, and molecular similarities [2-4]. As those tumors are rare, have been only 


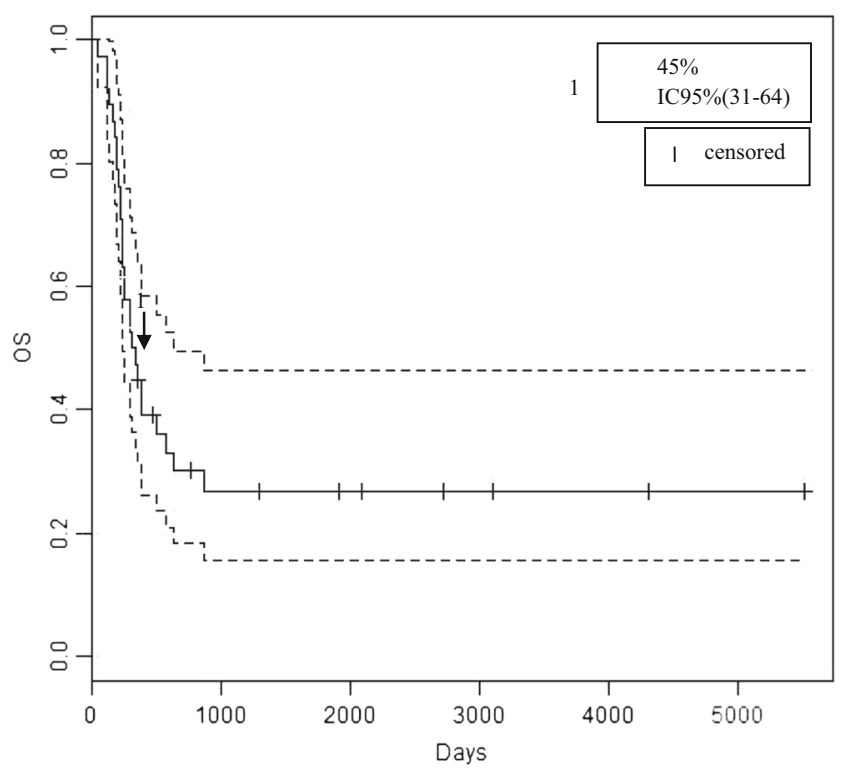

Fig. 1 Overall survival

recently described and diagnosis difficult to establish; there is only a limited number of publications concerning ETMR [2, $3,7,8]$. The series we report here represents the second largest series.

The patients' characteristics we report here appear similar to those reported in previous studies [2, 10]. ETMR mainly occurs in young children $[1,2,7]$, with a median of age at diagnosis of 31 months found in our cohort. The sex ratio in our series is 0.4 . This ratio is in line with the ratio reported by Picard et al. [10], where a sex ratio of 0.6 were found in the 29 included patients. As previously reported, the most frequent clinical sign is an increased intracranial pressure, which is commonly found in cerebral tumor in children [11-13]. Clinical signs at diagnosis are similar for all ETMR histological

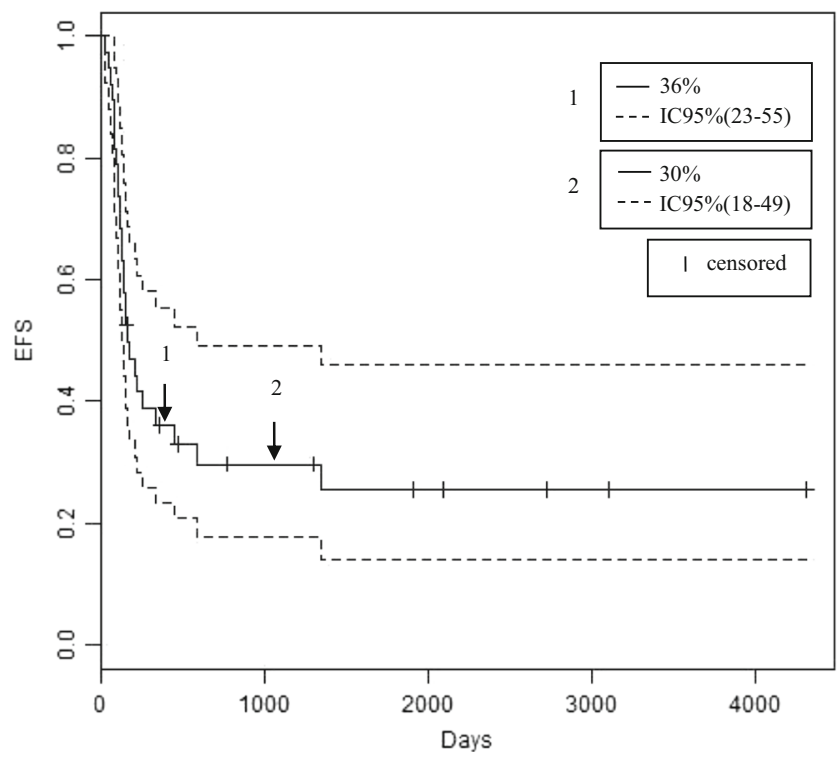

Fig. 2 Event-free survival variants [2]. Our results (Table 1) are consistent with these findings. Nevertheless, it should be noted that there is no ependymoblastoma in our series, probably due to the fact that the number of patients included in the cohort we report here is limited. A supratentorial location was found in the majority of the cases (66\%), as previously published [2]. We also report one patient with an ETMR localized to the spinal cord. This had never been reported before. Interestingly, metastasis was present at diagnosis in $18.9 \%$ of the children as reported by Korshunov et al. [2]. Metastasis were only evidenced using MRI but were never found on CSF pathological analysis at diagnosis. Of note, one case had a CSF positivity after second surgery.

The diagnosis of ETMR appears to be challenging, as illustrated by the fact that among the histopathological review we report here, there was a change in diagnosis in $16 \%$ cases (Table 2). The molecular diagnosis with LIN28A and the research of the gain of the chromosome 2 was performed in 11 patients. The amplification of the locus $19 \mathrm{q} 13.42$ was performed in 12 patients. LIN28A staining was positive in $100 \%$ of children, and $83 \%$ of children presented an amplification of the locus 19q13.42. Those results are in line with the data available in the literature and confirm that ETMR has a specific molecular signature. Therefore, immunohistochemistry for LIN28A and CGHarray for amplification of the locus $19 \mathrm{q} 13.42$ should be done routinely to secure the diagnosis.

One of the interests of the series we report here relies on the fact that the majority of the patients were treated homogenously according to the PNET-HR protocol [9]. Despite a multimodal therapy given in 30 patients, progressions were observed in all patients with a residual tumor during induction standard therapy or sequential high-dose therapy, and 26 of them died. Noteworthy, most of the patients presented either a local or both a local and distant relapse highlighting the aggressiveness of ETMR.

In the literature, radiotherapy was the only prognostic factor in univariate analysis [11]. This is confirmed by our findings which show that using both univariate and multivariate analyses, radiotherapy is associated with a better outcome. To the best of our knowledge, there is no other data on the prognostic factors available in the medical literature. Noteworthy, radiotherapy is given at the end of the treatment after chemotherapy which might select patient and introduce a bias. In the present series, we also found that a complete surgery and highdose chemotherapy were favorable prognostic factors. Indeed, out the 11 survivors, all but 2 children received high-dose chemotherapy. This is consistent with the experience of Dufour et al. [9] who reported the feasibility and effectiveness of high-dose chemotherapy ( 2 cycles of thiotepa) followed by conventional craniospinal radiotherapy with newly diagnosed high-risk medulloblastoma or supratentorial PNET. In their study, the 5-year overall survival was $85 \%$ for the whole 
cohort. Unfortunately, the impact of high-dose chemotherapy on long-term survival is difficult to extrapolate from the literature on ETMR due to small patient numbers. But, such aggressive chemotherapy, as in other embryonal central nervous system, could compensate for the avoidance or dose reduction in prophylactic craniospinal irradiation [14]. Such a strategy might lead to acceptable outcomes both in terms of toxicity and survival [15].

Patients with ETMR have a poor prognosis with an EFS and OS of, respectively, $36 \%$ CI $95 \%(23-55)$ and $45 \%$ CI $95 \%$ (31-64). In comparison, Korshunov et al. found a worst OS (14\%). This may be due to their treatment strategy which relies on chemotherapy and high-dose chemotherapy after surgery [4] but not on radiotherapy. Radiotherapy may therefore be recommended in children with ETMR. However, as radiotherapy potentially impairs neurocognitive function, especially in young children [16], and as children with ETMR are for the most of all less than 4 years old, benefits and longterm toxicities have to be balanced.

\section{Conclusion}

ETMR are rare cerebral tumors of the young child. A biologic diagnosis with LIN28A immuno-histochemistry and the search for amplification of the locus 19q13.42 should be systematically done to ensure diagnosis. Prognosis remains dismal, but complete surgical removal seems to be an important part of the treatment. Radiotherapy may be another crucial point of the treatment but has to be balanced with neurocognitive toxicity that can be caused in young children. Chemotherapy and maintenance chemotherapy may be useful, but their respective roles remain to be determined.

Conflict of interest The authors declare no conflict of interest.

\section{References}

1. Ceccom J, Bourdeaut F, Loukh N, Rigau V, Milin S, Takin R, et al. (2014) Embryonal tumor with multilayered rosettes: diagnostic tools update and review of the literature. Clin Neuropathol 33(1): $15-22$

2. Korshunov A, Sturm D, Ryzhova M, Hovestadt V, Gessi M, Jones DTW, et al. (2014) Embryonal tumor with abundant neuropil and true rosettes (ETANTR), ependymoblastoma, and medulloepithelioma share molecular similarity and comprise a single clinicopathological entity. Acta Neuropathol 128(2):279-289
3. Paulus W, Kleihues P (2010) Genetic profiling of CNS tumors extends histological classification. Acta Neuropathol 120(2):269 270

4. Korshunov A, Remke M, Gessi M, Ryzhova M, Hielscher T, Witt $\mathrm{H}$, et al. (2010) Focal genomic amplification at 19q13.42 comprises a powerful diagnostic marker for embryonal tumors with ependymoblastic rosettes. Acta Neuropathol 120(2):253-260

5. Korshunov A, Ryzhova M, Jones DTW, Northcott PA, van Sluis P, Volckmann R, et al. (2012) LIN28A immunoreactivity is a potent diagnostic marker of embryonal tumor with multilayered rosettes (ETMR). Acta Neuropathol 124(6):875-881

6. Louis DN, Ohgaki H, Wiestler OD, Cavenee WK, Burger PC, Jouvet A, et al. (2007) The 2007 WHO classification of tumours of the central nervous system. Acta Neuropathol 114(2):97-109

7. Eberhart CG, Brat DJ, Cohen KJ, Burger PC (2000) Pediatric neuroblastic brain tumors containing abundant neuropil and true rosettes. Pediatr Dev Pathol 3(4):346-352

8. Gessi M, Giangaspero F, Lauriola L, Gardiman M, Scheithauer BW, Halliday W, et al. (2009) Embryonal tumors with abundant neuropil and true rosettes: a distinctive CNS primitive neuroectodermal tumor. Am J Surg Pathol 33(2):211-217

9. Dufour C, Delisle M-B, Geoffray A, Laplanche A, Frappaz D, Icher C, et al. (2014) CT-003. Tandem high-dose chemotherapy with stem cell rescue followed by risk-adapted radiation in children with high-risk cerebral primitive neuroectodermal tumor: results of the prospective sfce-trial pnet $\mathrm{hr}+5$. Neuro-Oncology 16(suppl 1):i10 i13

10. Picard D, Miller S, Hawkins CE, Bouffet E, Rogers HA, Chan TSY, et al. (2012) Markers of survival and metastatic potential in childhood CNS primitive neuro-ectodermal brain tumours: an integrative genomic analysis. Lancet Oncol 13(8):838-848

11. Alexiou GA, Stefanaki K, Vartholomatos G, Sfakianos G, Prodromou N, Moschovi M (2013) Embryonal tumor with abundant neuropil and true rosettes: a systematic literature review and report of 2 new cases. J Child Neurol 28(12):1709-1715

12. Buccoliero AM, Castiglione F, Rossi Degl'Innocenti D, Franchi A, Paglierani M, Sanzo M, et al. (2010) Embryonal tumor with abundant neuropil and true rosettes: morphological, immunohistochemical, ultrastructural and molecular study of a case showing features of medulloepithelioma and areas of mesenchymal and epithelial differentiation. Neuropathology 30(1):84-91

13. Lafay-Cousin L, Hader W, Wei XC, Nordal R, Strother D, Hawkins C, et al. (2014) Post-chemotherapy maturation in supratentorial primitive neuroectodermal tumors. Brain Pathol 24(2):166-172

14. Odagiri K, Omura M, Hata M, Aida N, Niwa T, Goto H, et al. (2014) Treatment outcomes and late toxicities in patients with embryonal central nervous system tumors. Radiat Oncol 9:201

15. Kim SY, Sung KW, Hah JO, Yoo KH, Koo HH, Kang HJ, et al. (2010) Reduced-dose craniospinal radiotherapy followed by highdose chemotherapy and autologous stem cell rescue for children with newly diagnosed high-risk medulloblastoma or supratentorial primitive neuroectodermal tumor. Korean J Hematol 45(2):120 126

16. Padovani L, André N, Constine LS, Muracciole X (2012) Neurocognitive function after radiotherapy for paediatric brain tumours. Nat Rev Neurol 8(10):578-588 